\title{
Overview of the GIVD-registered databases
}

\author{
Falko Glöckler
}

\begin{abstract}
The contribution lists all 182 vegetation-plot databases registered in the Global Index of Vegetation-Plot Databases (GIVD) as of 12 July 2012. For each database, the GIVD ID, the proper name, and, where applicable, a descriptive subtitle, as well as the number of non-overlapping plots are given. The databases are arranged by their GIVD ID and grouped according to continents. For each database it is indicated whether it is presented with a Long Database Report, a Short Database Report, or not at all in this volume and the web link where up-to-date metadata are available.
\end{abstract}

Keywords: database report; ecoinformatics; Global Index of Vegetation-Plot Databases.

Abbreviations: GIVD ID = unique identifier in the Global Index of Vegetation-Plot Databases; LDR = Long Database Report; SDR = Short Database Report; URI = Uniform Resource Identifier.

\begin{tabular}{|c|c|c|c|c|c|}
\hline GIVD ID & Database: name - subtitle & $\begin{array}{l}\text { Non- } \\
\text { overlapping } \\
\text { plots }\end{array}$ & Report & Page & URI \\
\hline 00-00-001 & $\begin{array}{l}\text { ForestPlots.net - managing permanent plot information } \\
\text { across the tropics }\end{array}$ & 800 & LDR & 95 & http://www.givd.info/ID/00-00-001 \\
\hline 00-00-002 & $\begin{array}{l}\text { Tropical Ecology Assessment and Monitoring Network } \\
\text { (TEAM Network) }\end{array}$ & 94 & SDR & 287 & http://www.givd.info/ID/00-00-002 \\
\hline 00-00-003 & SALVIAS - the SALVIAS vegetation inventory database & 13661 & SDR & 288 & http://www.givd.info/ID/00-00-003 \\
\hline 00-RU-001 & Vegetation Database Forest of Southern Ural & 2270 & SDR & 289 & http://www.givd.info/ID/00-RU-001 \\
\hline 00-RU-002 & $\begin{array}{l}\text { Database of Masaryk University's Vegetation Research } \\
\text { in Siberia }\end{array}$ & 1157 & SDR & 290 & http://www.givd.info/ID/00-RU-002 \\
\hline 00-RU-003 & Database Meadows and Steppes of South Ural & 1363 & SDR & 291 & http://www.givd.info/ID/00-RU-003 \\
\hline 00-RU-004 & Database of South Ural Order Galietalia veri & 284 & No & - & http://www.givd.info/ID/00-RU-004 \\
\hline 00-RU-005 & Database of South Ural Order Arrhenatheretalia & 282 & No & - & http://www.givd.info/ID/00-RU-005 \\
\hline AF-00-001 & West African Vegetation Database & 12000 & LDR & 105 & http://www.givd.info/ID/AF-00-001 \\
\hline AF-00-003 & $\begin{array}{l}\text { BIOTA Southern Africa Biodiversity Observatories } \\
\text { Vegetation Database }\end{array}$ & 4083 & LDR & 111 & http://www.givd.info/ID/AF-00-003 \\
\hline AF-00-004 & Vegetation Database East Africa & 1850 & SDR & 292 & http://www.givd.info/ID/AF-00-004 \\
\hline AF-00-005 & $\begin{array}{l}\text { FLOTROP - a biogeographical database on rangeland } \\
\text { vegetation for northern tropical Africa }\end{array}$ & 34533 & SDR & 293 & http://www.givd.info/ID/AF-00-005 \\
\hline AF-00-006 & $\begin{array}{l}\text { SWEA-Dataveg - vegetation of small wetlands in East } \\
\text { Africa }\end{array}$ & 206 & SDR & 294 & http://www.givd.info/ID/AF-00-006 \\
\hline AF-00-007 & Namib Desert Region Vegetation Database & 10902 & SDR & 296 & http://www.givd.info/ID/AF-00-007 \\
\hline AF-MA-001 & Vegetation Database of Southern Morocco & 1790 & SDR & 297 & http://www.givd.info/ID/AF-MA-001 \\
\hline AF-NA-001 & National Phytosociological Database of Namibia & 11017 & SDR & 298 & http://www.givd.info/ID/AF-NA-001 \\
\hline AF-ZA-001 & National Vegetation Database of South Africa & 47466 & SDR & 299 & http://www.givd.info/ID/AF-ZA-001 \\
\hline AS-00-001 & Korean Forest Database & 2007 & SDR & 300 & http://www.givd.info/ID/AS-00-001 \\
\hline AS-00-002 & Vegetation Database of North Asia & 9000 & No & - & http://www.givd.info/ID/AS-00-002 \\
\hline AS-CN-001 & China Forest-Steppe Ecotone Database & 356 & SDR & 302 & http://www.givd.info/ID/AS-CN-001 \\
\hline AS-EG-001 & Vegetation Database of Sinai in Egypt & 1622 & SDR & 303 & http://www.givd.info/ID/AS-EG-001 \\
\hline AS-IL-001 & $\begin{array}{l}\text { Israel Nature and Parks Authority (INPA) Biological } \\
\text { Databases }\end{array}$ & 1000 & SDR & 304 & http://www.givd.info/ID/AS-IL-001 \\
\hline AS-IR-001 & Vegetation Database of Iran & 2000 & SDR & 305 & http://www.givd.info/ID/AS-IR-001 \\
\hline AS-IR-002 & Vegetation Database of Mountain Wetlands of Iran & 1000 & SDR & 306 & http://www.givd.info/ID/AS-IR-002 \\
\hline AS-IR-003 & Vegetation Database of the Hyrcanian Area & 100 & SDR & 307 & http://www.givd.info/ID/AS-IR-003 \\
\hline
\end{tabular}




\begin{tabular}{|c|c|c|c|c|c|}
\hline GIVD ID & Database: name - subtitle & $\begin{array}{l}\text { Non- } \\
\text { overlapping } \\
\text { plots }\end{array}$ & Report & Page & URI \\
\hline AS-JP-001 & Phytosociological Relevé Database of Japan (PRDB) & 39287 & SDR & 308 & http://www.givd.info/ID/AS-JP-001 \\
\hline AS-KG-001 & $\begin{array}{l}\text { Vegetation Database of South-Western Kyrgyzstan - } \\
\text { the walnut-wildfruit forests and alpine pastures }\end{array}$ & 698 & SDR & 309 & http://www.givd.info/ID/AS-KG-001 \\
\hline AS-KW-001 & Kuwait Vegetation Database & 202 & SDR & 310 & http://www.givd.info/ID/AS-KW-001 \\
\hline AS-RU-001 & $\begin{array}{l}\text { Wetland Vegetation Database of Baikal Siberia } \\
\text { (WETBS) }\end{array}$ & 2806 & SDR & 312 & http://www.givd.info/ID/AS-RU-001 \\
\hline AS-RU-002 & Database of Siberian Vegetation (DSV) & 30611 & SDR & 313 & http://www.givd.info/ID/AS-RU-002 \\
\hline AS-TR-001 & $\begin{array}{l}\text { Vegetation Database of the Grassland Communities in } \\
\text { Anatolia }\end{array}$ & 1878 & SDR & 314 & http://www.givd.info/ID/AS-TR-001 \\
\hline AS-TR-002 & Vegetation Database of Oak Communities in Turkey & 1182 & SDR & 315 & http://www.givd.info/ID/AS-TR-002 \\
\hline AS-TW-001 & National Vegetation Database of Taiwan & 8035 & No & - & http://www.givd.info/ID/AS-TW-001 \\
\hline AS-YE-001 & Socotra Vegetation Database & 396 & SDR & 315 & http://www.givd.info/ID/AS-YE-001 \\
\hline AU-AU-001 & $\begin{array}{l}\text { PPBio Australasia Long Term Ecological Research } \\
\text { Sites }\end{array}$ & 99 & SDR & 316 & http://www.givd.info/ID/AU-AU-001 \\
\hline AU-NZ-001 & New Zealand National Vegetation Databank & 77000 & SDR & 318 & http://www.givd.info/ID/AU-NZ-001 \\
\hline EU-00-002 & $\begin{array}{l}\text { Database Dry Grasslands in the Nordic and Baltic } \\
\text { Region }\end{array}$ & 7675 & SDR & 319 & http://www.givd.info/ID/EU-00-002 \\
\hline EU-00-003 & $\begin{array}{l}\text { Database Species-Area Relationships in Palaearctic } \\
\text { Grasslands }\end{array}$ & 727 & SDR & 321 & http://www.givd.info/ID/EU-00-003 \\
\hline EU-00-004 & $\begin{array}{l}\text { Iberian and Macaronesian Vegetation Information } \\
\text { System (SIVIM) }\end{array}$ & 125000 & SDR & 323 & http://www.givd.info/ID/EU-00-004 \\
\hline EU-00-005 & $\begin{array}{l}\text { Vegetation Database Mulgedio-Aconitetea and Related } \\
\text { Vegetation Types }\end{array}$ & 2852 & SDR & 324 & http://www.givd.info/ID/EU-00-005 \\
\hline EU-00-006 & $\begin{array}{l}\text { Ecological Conditions Database }(E C) \text { - vegetation } \\
\text { relevés and soil measurements in the Netherlands }\end{array}$ & 8130 & LDR & 125 & http://www.givd.info/ID/EU-00-006 \\
\hline EU-00-007 & VIOLETEA - heavy metal grasslands & 656 & SDR & 325 & http://www.givd.info/ID/EU-00-007 \\
\hline EU-00-008 & $\begin{array}{l}\text { Vegetation Database of Deciduous Forests on Acidic } \\
\text { Soils in NW Europe }\end{array}$ & 4437 & SDR & 326 & http://www.givd.info/ID/EU-00-008 \\
\hline EU-00-009 & $\begin{array}{l}\text { Vegetation Database of the Upper Rhine Alluvial Plain } \\
\text { Forests }\end{array}$ & 1100 & SDR & 327 & http://www.givd.info/ID/EU-00-009 \\
\hline EU-00-011 & $\begin{array}{l}\text { Vegetation-Plot Database of the University of the } \\
\text { Basque Country (BIOVEG) }\end{array}$ & 20172 & SDR & 328 & http://www.givd.info/ID/EU-00-011 \\
\hline EU-00-012 & Beech Forest Vegetation Database of SE Balkan & 5952 & SDR & 329 & http://www.givd.info/ID/EU-00-012 \\
\hline EU-00-013 & Balkan Dry Grasslands Database & 4520 & SDR & 330 & http://www.givd.info/ID/EU-00-013 \\
\hline EU-00-014 & $\begin{array}{l}\text { The Alps Vegetation Database - a geo-referenced } \\
\text { community-level archive of all terrestrial plants } \\
\text { occurring in the Alps }\end{array}$ & 24474 & SDR & 331 & http://www.givd.info/ID/EU-00-014 \\
\hline EU-AT-001 & Austrian Vegetation Database & 42000 & SDR & 333 & http://www.givd.info/ID/EU-AT-001 \\
\hline EU-AZ-001 & $\begin{array}{l}\text { Vegetation Database of the Shahdag Region, } \\
\text { Azerbaijan }\end{array}$ & 222 & SDR & 334 & http://www.givd.info/ID/EU-AZ-001 \\
\hline EU-BE-001 & $\begin{array}{l}\text { VLAVEDAT - the vegetation database of Flanders } \\
\text { (northern Belgium) }\end{array}$ & 26180 & LDR & 133 & http://www.givd.info/ID/EU-BE-001 \\
\hline EU-BG-001 & Bulgarian Vegetation Database & 5901 & LDR & 141 & http://www.givd.info/ID/EU-BG-001 \\
\hline EU-BY-001 & Belarus Peatland Restoration Project Database & 900 & SDR & 335 & http://www.givd.info/ID/EU-BY-001 \\
\hline EU-CH-001 & $\begin{array}{l}\text { Permanent.Plot.ch - a database for Swiss permanent } \\
\text { vegetation plots }\end{array}$ & 919 & SDR & 337 & http://www.givd.info/ID/EU-CH-001 \\
\hline EU-CH-002 & Swiss Biodiversity Monitoring BDM (Z9 Plants) & 1600 & SDR & 338 & http://www.givd.info/ID/EU-CH-002 \\
\hline EU-CH-004 & National Inventory of Swiss Bryophytes (NISM) & 1237 & SDR & 339 & http://www.givd.info/ID/EU-CH-004 \\
\hline EU-CH-005 & Swiss Forest Vegetation Database & 14800 & SDR & 340 & http://www.givd.info/ID/EU-CH-005 \\
\hline EU-CH-006 & Database Dry Meadows and Pastures of Switzerland & 13724 & SDR & 341 & http://www.givd.info/ID/EU-CH-006 \\
\hline EU-CH-007 & $\begin{array}{l}\text { Swiss Mire Monitoring - Wirkungskontrolle Moorschutz } \\
\text { Schweiz }\end{array}$ & 24015 & No & - & http://www.givd.info/ID/EU-CH-007 \\
\hline EU-CH-008 & Phytobase C2005 & 1241 & SDR & 342 & http://www.givd.info/ID/EU-CH-008 \\
\hline EU-CH-009 & Phytobase S2008 & 1314 & SDR & 343 & http://www.givd.info/ID/EU-CH-009 \\
\hline EU-CH-010 & Swiss Biodiversity Monitoring BDM (Z9 Mosses) & 1600 & SDR & 344 & http://www.givd.info/ID/EU-CH-010 \\
\hline EU-CZ-001 & Czech National Phytosociological Database & 99586 & SDR & 345 & http://www.givd.info/ID/EU-CZ-001 \\
\hline EU-CZ-002 & Database of Czech Forest Classification System & 32387 & SDR & 346 & http://www.givd.info/ID/EU-CZ-002 \\
\hline EU-DE-001 & $\begin{array}{l}\text { VegMV - the vegetation database of Mecklenburg- } \\
\text { Vorpommern }\end{array}$ & 53842 & LDR & 149 & http://www.givd.info/ID/EU-DE-001 \\
\hline
\end{tabular}




\begin{tabular}{|c|c|c|c|c|c|}
\hline GIVD ID & Database: name - subtitle & $\begin{array}{l}\text { Non- } \\
\text { overlapping } \\
\text { plots }\end{array}$ & Report & Page & URI \\
\hline EU-DE-002 & $\begin{array}{l}\text { BERGWALD - the vegetation database of mountain } \\
\text { forests in the Bavarian Alps }\end{array}$ & 4934 & LDR & 161 & http://www.givd.info/ID/EU-DE-002 \\
\hline EU-DE-003 & $\begin{array}{l}\text { WINALPecobase - ecological database of mountain } \\
\text { forests in the Bavarian Alps }\end{array}$ & 1505 & LDR & 167 & http://www.givd.info/ID/EU-DE-003 \\
\hline EU-DE-004 & Database Polygono-Poetea annuae of Germany & 584 & SDR & 347 & http://www.givd.info/ID/EU-DE-004 \\
\hline EU-DE-005 & $\begin{array}{l}\text { Database Temperate Deciduous Forests of the Elbe- } \\
\text { Weser Region }\end{array}$ & 415 & SDR & 348 & http://www.givd.info/ID/EU-DE-005 \\
\hline EU-DE-006 & $\begin{array}{l}\text { Database Temperate Deciduous Forests of the Prignitz } \\
\text { Region }\end{array}$ & 232 & SDR & 349 & http://www.givd.info/ID/EU-DE-006 \\
\hline EU-DE-007 & $\begin{array}{l}\text { Database Temperate Deciduous Forests of the } \\
\text { Uckermark Region }\end{array}$ & 500 & SDR & 350 & http://www.givd.info/ID/EU-DE-007 \\
\hline EU-DE-008 & Cytisus_SFB299 & 220 & No & - & http://www.givd.info/ID/EU-DE-008 \\
\hline EU-DE-009 & $\begin{array}{l}\text { BioChangeMeadows - German meadows in the 1950s, } \\
1990 \text { s and in } 2008\end{array}$ & 1092 & SDR & 351 & http://www.givd.info/ID/EU-DE-009 \\
\hline EU-DE-010 & Vegetation Database Forests of the Oldenburg Region & 1522 & SDR & 352 & http://www.givd.info/ID/EU-DE-010 \\
\hline EU-DE-011 & $\begin{array}{l}\text { Vegetation Database Forests and Grasslands of the } \\
\text { Lower Werra Region }\end{array}$ & 565 & SDR & 353 & http://www.givd.info/ID/EU-DE-011 \\
\hline EU-DE-012 & Vegetation Database Nardus Swards of Germany & 419 & SDR & 354 & http://www.givd.info/ID/EU-DE-012 \\
\hline EU-DE-013 & $\begin{array}{l}\text { VegetWeb - the national online-repository of vegetation } \\
\text { plots from Germany }\end{array}$ & 26692 & LDR & 173 & http://www.givd.info/ID/EU-DE-013 \\
\hline EU-DE-014 & German Vegetation Reference Database (GVRD) & 111928 & SDR & 355 & http://www.givd.info/ID/EU-DE-014 \\
\hline EU-DE-015 & $\begin{array}{l}\text { Vegetation Database of Successional Permanent Plots } \\
\text { in Göttingen }\end{array}$ & 23 & SDR & 356 & http://www.givd.info/ID/EU-DE-015 \\
\hline EU-DE-016 & $\begin{array}{l}\text { Vegetation Database of Strict Forest Reserves in NW- } \\
\text { Germany }\end{array}$ & 3600 & SDR & 357 & http://www.givd.info/ID/EU-DE-016 \\
\hline EU-DE-017 & $\begin{array}{l}\text { Database Temperate Deciduous and Coniferous } \\
\text { Forests of the Solling Hills }\end{array}$ & 2000 & SDR & 358 & http://www.givd.info/ID/EU-DE-017 \\
\hline EU-DE-018 & $\begin{array}{l}\text { Vegetation Database of Temperate Deciduous Forests } \\
\text { of the Göttinger Wald }\end{array}$ & 350 & SDR & 359 & http://www.givd.info/ID/EU-DE-018 \\
\hline EU-DE-019 & $\begin{array}{l}\text { Vegetation Database of Pine Forests on Acidic Soils in } \\
\text { Germany }\end{array}$ & 3327 & SDR & 360 & http://www.givd.info/ID/EU-DE-019 \\
\hline EU-DE-020 & Dry Grassland Database of Germany & 0 & No & - & http://www.givd.info/ID/EU-DE-020 \\
\hline EU-DE-021 & Vegetation Database Main-Kinzig + Bergland & 1562 & SDR & 361 & http://www.givd.info/ID/EU-DE-021 \\
\hline EU-DE-022 & Vegetation Database Frankenalb & 2500 & SDR & 362 & http://www.givd.info/ID/EU-DE-022 \\
\hline EU-DE-023 & Post-Mining Vegetation Database Eastern Germany & 3247 & SDR & 363 & http://www.givd.info/ID/EU-DE-023 \\
\hline EU-DE-024 & Disturbances and Biodiversity in the Fichtelgebirge & 524 & SDR & 364 & http://www.givd.info/ID/EU-DE-024 \\
\hline EU-DE-025 & $\begin{array}{l}\text { Disturbances and Biodiversity at Grafenwöhr Training } \\
\text { Area }\end{array}$ & 595 & SDR & 365 & http://www.givd.info/ID/EU-DE-025 \\
\hline EU-DE-026 & Vaccinio-Pinetea Database & 2000 & No & - & http://www.givd.info/ID/EU-DE-026 \\
\hline EU-DE-027 & $\begin{array}{l}\text { BioChangeFields - vegetation database of arable plant } \\
\text { communties in Central Germany }\end{array}$ & 1176 & SDR & 366 & http://www.givd.info/ID/EU-DE-027 \\
\hline EU-DE-028 & AuVeg - a database of German floodplain vegetation & 5180 & SDR & 367 & http://www.givd.info/ID/EU-DE-028 \\
\hline EU-DE-029 & $\begin{array}{l}\text { Database of German North Sea Salt Marshes - } \\
\text { Changes in Vegetation and Elevation }\end{array}$ & 423 & SDR & 368 & http://www.givd.info/ID/EU-DE-029 \\
\hline EU-DE-030 & $\begin{array}{l}\text { Database of German North Sea Salt Marshes - } \\
\text { Vegetation and Elevation }\end{array}$ & 2691 & SDR & 370 & http://www.givd.info/ID/EU-DE-030 \\
\hline EU-DE-031 & Lübeck Beech Forest Biodiversity Plots & 86 & SDR & 371 & http://www.givd.info/ID/EU-DE-031 \\
\hline EU-DE-032 & TMAP Wadden Sea Sedimentation Database & 144 & SDR & 372 & http://www.givd.info/ID/EU-DE-032 \\
\hline EU-DE-033 & TMAP Wadden Sea Vegetation Database & 249 & SDR & 373 & http://www.givd.info/ID/EU-DE-033 \\
\hline EU-DE-034 & Dry Grassland Central Germany Database & 2500 & SDR & 374 & http://www.givd.info/ID/EU-DE-034 \\
\hline EU-DK-001 & $\begin{array}{l}\text { NATURDATA.DK - Danish monitoring program of } \\
\text { vegetation and chemical plant and soil data from non- } \\
\text { forested terrestrial habitat types }\end{array}$ & 1200 & SDR & 375 & http://www.givd.info/ID/EU-DK-001 \\
\hline EU-EE-001 & Estonian Vegetation Database & 1430 & SDR & 376 & http://www.givd.info/ID/EU-EE-001 \\
\hline EU-FI-001 & Vegetation Database of Eurasian Tundra & 1300 & SDR & 377 & http://www.givd.info/ID/EU-FI-001 \\
\hline EU-FR-001 & $\begin{array}{l}\text { Vigie-flore - a nationwide monitoring programme for } \\
\text { common plant species and plant communities in France }\end{array}$ & 165 & SDR & 378 & http://www.givd.info/ID/EU-FR-001 \\
\hline EU-FR-002 & FLOREM - a floristic database on moutain ecosystems & 2000 & SDR & 380 & http://www.givd.info/ID/EU-FR-002 \\
\hline EU-FR-003 & $\begin{array}{l}\text { SOPHY - basis of plant socio-ecology and } \\
\text { phytoclimatology in France }\end{array}$ & 212244 & LDR & 177 & http://www.givd.info/ID/EU-FR-003 \\
\hline
\end{tabular}




\begin{tabular}{|c|c|c|c|c|c|}
\hline GIVD ID & Database: name - subtitle & $\begin{array}{c}\text { Non- } \\
\text { overlapping } \\
\text { plots }\end{array}$ & Report & Page & URI \\
\hline EU-GB-001 & UK National Vegetation Classification Database & 35000 & SDR & 381 & http://www.givd.info/ID/EU-GB-001 \\
\hline EU-GB-002 & $\begin{array}{l}\text { UK Environmental Change Network - Vegetation } \\
\text { Database }\end{array}$ & 3783 & SDR & 382 & http://www.givd.info/ID/EU-GB-002 \\
\hline EU-GB-003 & $\begin{array}{l}\text { Vegetation Database of Great Britain: Countryside } \\
\text { Survey }\end{array}$ & 49165 & SDR & 383 & http://www.givd.info/ID/EU-GB-003 \\
\hline EU-GR-001 & $\mathrm{KRITI}$ - the vegetation of Crete database & 6500 & SDR & 384 & http://www.givd.info/ID/EU-GR-001 \\
\hline EU-GR-002 & Greek Woodland Vegetation Database & 3500 & SDR & 385 & http://www.givd.info/ID/EU-GR-002 \\
\hline EU-GR-003 & $\begin{array}{l}\text { Vegetation Database Isoeto-Nano-Juncetea in Greece } \\
\text { and the Aegean Region }\end{array}$ & 300 & SDR & 386 & http://www.givd.info/ID/EU-GR-003 \\
\hline EU-GR-004 & Segetal Weed Vegetation Database of Greece & 200 & SDR & 387 & http://www.givd.info/ID/EU-GR-004 \\
\hline EU-GR-005 & Hellenic Natura 2000 Vegetation Database (HelNatVeg) & 13862 & SDR & 388 & http://www.givd.info/ID/EU-GR-005 \\
\hline EU-GR-006 & Hellenic Woodland Database & 4571 & SDR & 389 & http://www.givd.info/ID/EU-GR-006 \\
\hline EU-GR-007 & Hellenic Beech Forests Database (Hell-Beech-DB) & 1404 & SDR & 390 & http://www.givd.info/ID/EU-GR-007 \\
\hline EU-HR-001 & $\begin{array}{l}\text { Phytosociological Database of Non-Forest Vegetation in } \\
\text { Croatia }\end{array}$ & 5728 & SDR & 391 & http://www.givd.info/ID/EU-HR-001 \\
\hline EU-HU-001 & Regional Vegetation Database of Kiskunsag & 605 & SDR & 392 & http://www.givd.info/ID/EU-HU-001 \\
\hline EU-HU-002 & Long-term Database of Sandy Grassland of Fulophaza & 200 & SDR & 393 & http://www.givd.info/ID/EU-HU-002 \\
\hline EU-HU-003 & CoenoDat Hungarian Phytosociological Database & 11000 & SDR & 394 & http://www.givd.info/ID/EU-HU-003 \\
\hline EU-IE-001 & Irish Vegetation Database & 26412 & SDR & 395 & http://www.givd.info/ID/EU-IE-001 \\
\hline EU-IT-001 & $\begin{array}{l}\text { Vegltaly - toward an Italian national vegetation } \\
\text { database }\end{array}$ & 11120 & LDR & 185 & http://www.givd.info/ID/EU-IT-001 \\
\hline EU-IT-002 & Vegetation Database of the Cilento National Park & 2289 & SDR & 396 & http://www.givd.info/ID/EU-IT-002 \\
\hline EU-IT-003 & Lucanian Vegetation Database & 1810 & SDR & 397 & http://www.givd.info/ID/EU-IT-003 \\
\hline EU-IT-004 & Dry Grasslands Database of Central Italy & 762 & SDR & 398 & http://www.givd.info/ID/EU-IT-004 \\
\hline EU-IT-005 & $\begin{array}{l}\text { VegDunes - A coastal dune vegetation database for the } \\
\text { analysis of Italian EU habitats }\end{array}$ & 2666 & LDR & 191 & http://www.givd.info/ID/EU-IT-005 \\
\hline EU-IT-006 & Deciduous Oak Forests Database of Italian Peninsula & 1600 & SDR & 399 & http://www.givd.info/ID/EU-IT-006 \\
\hline EU-IT-007 & Macrophytes of Italian Volcanic Lakes Database & 1776 & SDR & 401 & http://www.givd.info/ID/EU-IT-007 \\
\hline EU-IT-008 & EVSItalia Database Dry Grasslands & 963 & SDR & 402 & http://www.givd.info/ID/EU-IT-008 \\
\hline EU-IT-009 & $\begin{array}{l}\text { EVSItalia Database Broadleaved Deciduous } \\
\text { Submediterranean Forests }\end{array}$ & 733 & SDR & 403 & http://www.givd.info/ID/EU-IT-009 \\
\hline EU-IT-010 & Italian National Vegetation Database (BVN/ISPRA) & 4400 & SDR & 404 & http://www.givd.info/ID/EU-IT-010 \\
\hline EU-IT-011 & $\begin{array}{l}\text { EVSItalia Database Broadleaved Temperate Deciduous } \\
\text { Forests }\end{array}$ & 1092 & SDR & 405 & http://www.givd.info/ID/EU-IT-011 \\
\hline EU-IT-013 & $\begin{array}{l}\text { EVSItalia Database Eastern Alps - from beech forest to } \\
\text { alpine grasslands (Dolomites) }\end{array}$ & 639 & No & - & http://www.givd.info/ID/EU-IT-013 \\
\hline EU-IT-014 & EVSItalia Database Evergreen Mediterranean Forests & 1340 & SDR & 406 & http://www.givd.info/ID/EU-IT-014 \\
\hline EU-IT-015 & $\begin{array}{l}\text { EVSItalia Database Halophytic Littoral and Inland } \\
\text { Vegetation }\end{array}$ & 349 & No & - & http://www.givd.info/ID/EU-IT-015 \\
\hline EU-IT-016 & EVSItalia Database Mires \& Wetlands of Italy & 263 & SDR & 407 & http://www.givd.info/ID/EU-IT-016 \\
\hline EU-IT-017 & Vegetation Databases of Sicily & 0 & No & - & http://www.givd.info/ID/EU-IT-017 \\
\hline EU-IT-018 & EVSItalia Database HABITAT OF ITALY & 5441 & SDR & 408 & http://www.givd.info/ID/EU-IT-018 \\
\hline EU-LV-001 & Semi-natural Grassland Vegetation Database of Latvia & 2165 & SDR & 409 & http://www.givd.info/ID/EU-LV-001 \\
\hline EU-LV-002 & Mire Vegetation Database of Latvia & 2083 & SDR & 410 & http://www.givd.info/ID/EU-LV-002 \\
\hline EU-NL-001 & Dutch National Vegetation Database & 600000 & LDR & 201 & http://www.givd.info/ID/EU-NL-001 \\
\hline EU-NL-002 & Vegetation Database of Dutch Road Verges & 2552 & No & - & http://www.givd.info/ID/EU-NL-002 \\
\hline EU-PL-001 & Polish Vegetation Database & 43261 & SDR & 411 & http://www.givd.info/ID/EU-PL-001 \\
\hline EU-RO-001 & $\begin{array}{l}\text { Vegetation Database of Dry Grasslands in the } \\
\text { Southeast Romania }\end{array}$ & 2500 & SDR & 412 & http://www.givd.info/ID/EU-RO-001 \\
\hline EU-RO-002 & $\begin{array}{l}\text { Vegetation Database of the Dry Grasslands from the } \\
\text { Transylvanian Basin }\end{array}$ & 1467 & SDR & 413 & http://www.givd.info/ID/EU-RO-002 \\
\hline EU-RO-003 & $\begin{array}{l}\text { Oak-Hornbeam Forest Database of the Transylvanian } \\
\text { Basin }\end{array}$ & 524 & SDR & 414 & http://www.givd.info/ID/EU-RO-003 \\
\hline EU-RO-004 & Database Beech Forests from Romanian Carpathians & 816 & SDR & 415 & http://www.givd.info/ID/EU-RO-004 \\
\hline EU-RO-005 & Vegetation Database of Oak Forests of Romania & 4398 & SDR & 416 & http://www.givd.info/ID/EU-RO-005 \\
\hline EU-RS-001 & Database of Halophytic Vegetation in Serbia & 1026 & SDR & 417 & http://www.givd.info/ID/EU-RS-001 \\
\hline EU-RS-002 & Vegetation Database Grassland Vegetation of Serbia & 4252 & SDR & 418 & http://www.givd.info/ID/EU-RS-002 \\
\hline EU-RU-002 & Lower Volga Valley Phytosociological Database & 13280 & SDR & 419 & http://www.givd.info/ID/EU-RU-002 \\
\hline
\end{tabular}




\begin{tabular}{|c|c|c|c|c|c|}
\hline GIVD ID & Database: name - subtitle & $\begin{array}{l}\text { Non- } \\
\text { overlapping } \\
\text { plots }\end{array}$ & Report & Page & URI \\
\hline EU-RU-003 & $\begin{array}{l}\text { Vegetation Database of the Volga and the Ural Rivers } \\
\text { Basins }\end{array}$ & 4500 & SDR & 420 & http://www.givd.info/ID/EU-RU-003 \\
\hline EU-RU-004 & $\begin{array}{l}\text { Coastal Vegetation Database of North-Western Seas of } \\
\text { Russia }\end{array}$ & 1529 & SDR & 422 & http://www.givd.info/ID/EU-RU-004 \\
\hline EU-RU-005 & $\begin{array}{l}\text { Coastal Vegetation Database of Southern Seas of } \\
\text { Russia }\end{array}$ & 1936 & SDR & 423 & http://www.givd.info/ID/EU-RU-005 \\
\hline EU-RU-006 & Moscow Region Forest Vegetation Database & 750 & SDR & 424 & http://www.givd.info/ID/EU-RU-006 \\
\hline EU-RU-007 & Database Biodiversity of Murmansk Region & 350 & SDR & 425 & http://www.givd.info/ID/EU-RU-007 \\
\hline EU-RU-008 & Vegetation Database of Sudost-Desna Interfluve Area & 2000 & SDR & 426 & http://www.givd.info/ID/EU-RU-008 \\
\hline EU-RU-009 & Curonian Spit Biodiversity Plots & 130 & SDR & 427 & http://www.givd.info/ID/EU-RU-009 \\
\hline EU-SI-001 & Vegetation Database of Slovenia & 15773 & SDR & 428 & http://www.givd.info/ID/EU-SI-001 \\
\hline EU-SK-001 & Slovak Vegetation Database & 51581 & SDR & 429 & http://www.givd.info/ID/EU-SK-001 \\
\hline EU-UA-001 & Ukrainian Grasslands Database & 3181 & SDR & 430 & http://www.givd.info/ID/EU-UA-001 \\
\hline NA-00-002 & Tree Biodiversity Network (BIOTREE-NET) & 2019 & LDR & 211 & http://www.givd.info/ID/NA-00-002 \\
\hline NA-CA-001 & $\begin{array}{l}\text { Database of Forest Understory Vegetation, British } \\
\text { Columbia - vascular plants and bryophytes in old- } \\
\text { growth and clear-cut forests, Vancouver Island }\end{array}$ & 72 & SDR & 431 & http://www.givd.info/ID/NA-CA-001 \\
\hline NA-CA-002 & Vegetation Database of Québec (MRNF) & 28425 & SDR & 432 & http://www.givd.info/ID/NA-CA-002 \\
\hline NA-US-001 & $\begin{array}{l}\text { Forest Inventory and Analysis Database of the United } \\
\text { States of America (FIA) }\end{array}$ & 538428 & LDR & 225 & http://www.givd.info/ID/NA-US-001 \\
\hline NA-US-002 & $\begin{array}{l}\text { VegBank - the vegetation-plot archive of the Ecological } \\
\text { Society of America }\end{array}$ & 22629 & LDR & 233 & http://www.givd.info/ID/NA-US-002 \\
\hline NA-US-003 & Jasper Ridge Woody Plant Community Database & 44 & SDR & 433 & http://www.givd.info/ID/NA-US-003 \\
\hline NA-US-004 & $\begin{array}{l}\text { Thirty Years of Permanent Vegetation Plots, Mount St. } \\
\text { Helens, Washington }\end{array}$ & 92 & SDR & 434 & http://www.givd.info/ID/NA-US-004 \\
\hline NA-US-005 & $\begin{array}{l}\text { Santa Rita Experimental Range Long Term Transect } \\
\text { Database }\end{array}$ & 132 & SDR & 435 & http://www.givd.info/ID/NA-US-005 \\
\hline NA-US-006 & Carolina Vegetation Survey & 8283 & LDR & 243 & http://www.givd.info/ID/NA-US-006 \\
\hline NA-US-007 & $\begin{array}{l}\text { FIADB Vegetation Diversity and Structure Indicator } \\
\text { (VEG) }\end{array}$ & 2564 & SDR & 436 & http://www.givd.info/ID/NA-US-007 \\
\hline NA-US-008 & $\begin{array}{l}\text { Wisconsin Vegetation Database - plant community } \\
\text { survey and resurvey data from the Wisconsin Plant } \\
\text { Ecology Laboratory }\end{array}$ & 2582 & LDR & 255 & http://www.givd.info/ID/NA-US-008 \\
\hline NA-US-009 & $\begin{array}{l}\text { Vegetation Database of Observatory Woods, Wisconsin } \\
\text { - mapped vegetation }\end{array}$ & 38 & SDR & 437 & http://www.givd.info/ID/NA-US-009 \\
\hline NA-US-011 & $\begin{array}{l}\text { Vegetation Database of Central New Mexico - desert } \\
\text { grass- and shrubland net-primary production quadrat } \\
\text { data }\end{array}$ & 120 & SDR & 438 & http://www.givd.info/ID/NA-US-011 \\
\hline NA-US-012 & $\begin{array}{l}\text { Epiphytic Macrolichen Community Composition } \\
\text { Database - epiphytic lichen synusiae in forested areas } \\
\text { of the US }\end{array}$ & 4941 & SDR & 439 & http://www.givd.info/ID/NA-US-012 \\
\hline NA-US-013 & Natural Heritage Vegetation Database for West Virginia & 3896 & SDR & 440 & http://www.givd.info/ID/NA-US-013 \\
\hline SA-00-001 & $\begin{array}{l}\text { Database Ephemeral Wetland Vegetation in Extra- and } \\
\text { Oro-Tropical South America }\end{array}$ & 514 & SDR & 441 & http://www.givd.info/ID/SA-00-001 \\
\hline SA-BO-001 & Project Database of Bolivian Ecoregions & 118 & SDR & 442 & http://www.givd.info/ID/SA-BO-001 \\
\hline SA-BR-001 & $\begin{array}{l}\text { Brazilian Progam for Biodiversity Research (PPBio) } \\
\text { Information System }\end{array}$ & 1843 & LDR & 265 & http://www.givd.info/ID/SA-BR-001 \\
\hline SA-CL-001 & $\begin{array}{l}\text { CL-Dataveg - a database of Chilean grassland } \\
\text { vegetation }\end{array}$ & 650 & SDR & 443 & http://www.givd.info/ID/SA-CL-001 \\
\hline SA-CO-001 & $\begin{array}{l}\text { Colombian Páramo Vegetation Database (CPVD) - the } \\
\text { database on high Andean páramo vegetation in } \\
\text { Colombia }\end{array}$ & 800 & LDR & 275 & http://www.givd.info/ID/SA-CO-001 \\
\hline SA-CO-002 & $\begin{array}{l}\text { Vegetation Database of the Colombian Caribbean } \\
\text { Region }\end{array}$ & 320 & SDR & 444 & http://www.givd.info/ID/SA-CO-002 \\
\hline SA-EC-001 & Ecuador Forest Plot Database & 230 & SDR & 446 & http://www.givd.info/ID/SA-EC-001 \\
\hline SA-VE-001 & Vegetation Database Guaramacal, Andes, Venezuela & 44 & SDR & 447 & http://www.givd.info/ID/SA-VE-001 \\
\hline
\end{tabular}




\section{Falko Glöckler}

(falko.gloeckler@gmx.de)

Biodiversity Informatics, Global

Biodiversity Information Facility

(GBIF), Natural History Museum

Berlin

Invalidenstr. 43

10115 Berlin, GERMANY 